PHYSICAL REVIEW A, VOLUME 64, 019903(E)

\title{
Erratum: Parameter estimation in quantum optics [Phys. Rev. A 62, 023815 (2000)]
}

G. Mauro D'Ariano, Matteo G. A. Paris, and Massimiliano F. Sacchi

(Published 31 May 2001)

DOI: 10.1103/PhysRevA.64.019903

PACS number(s): 42.50.Ar, 99.10.+g

The probability distribution in Eq. (30) of the original paper should read

$$
p_{\eta}(x)=\frac{1}{\sqrt{2 \pi \Delta^{2}}} \exp \left[-\frac{\left(x-\sqrt{\eta} x_{0}\right)^{2}}{2 \Delta^{2}}\right]
$$

such that the resulting maximum likelihood estimate (MLE) for the quantum efficiency $\eta$, i.e., $\eta_{M L}=\arg \max L(\eta)$, is given by the (unique) solution in $[0,1]$ of the algebraic equation

$$
\left(1-\eta+e^{-2 r}\right)\left[1-4 x_{0}\left(x_{0}-\frac{\bar{x}}{\sqrt{\eta}}\right)\right]=4\left(\overline{x^{2}}+\eta x_{0}^{2}-2 \sqrt{\eta} x_{0} \bar{x}\right) .
$$

Figure 5 and the physical conclusions remain unaltered, whereas Eq. (32) of the original paper is no longer valid. 